\title{
UTILIZAÇÃO DA LÓGICA FUZZY PARA OTIMIZAR APLICAÇÃO DE FERTILIZANTES NO RABANETE
}

\author{
USE OF FUZZY LOGIC TO OPTIMIZE FERTILIZER APPLICATION IN THE RADISH ${ }^{\circ}$ \\ USO DE LA LÓGICA FUZZY PARA OPTIMIZAR LA APLICACIÓN DE FERTILIZANTES \\ EN EL RÁBANO ${ }^{\circ}$
}

Recebido em: 20/07/2021 - Aprovado em: 08/09/2021 - Publicado em: 01/10/2021

doi) http://dx.doi.org/10.18011/bioeng2021v15n2p270-282

Emmanuel Zullo Godinho' (emmanuel.godinho@unesp.br)

Fernando de Lima Caneppele ${ }^{2}$ (caneppele@usp.br)

Hélio Vagner Gasparotto' (hélio.vagner@unesp.br)

\footnotetext{
1 Universidade Estadual Paulista. Botucatu, SP, Brasil.

2 Universidade de São Paulo. Pirassununga, SP, Brasil.
}

\section{RESUMO}

O objetivo deste estudo foi desenvolver um modelo baseado em regras difusas para avaliar os efeitos causados pela variação nas dosagens de dois fertilizantes de solo (mineral e orgânico) no diâmetro de raiz. A lógica difusa é um método que apresenta uma linguagem, mais apropriada ao dia a dia, como está meio nublado o céu. Para as variáveis de input deste sistema foi utilizado o fertilizante mineral e um orgânico, para o output o diâmetro de raiz, em $\mathrm{cm}$. Após a otimização das regras de entrada, pode-se constatar que para a aplicação dos fertilizantes (mineral e orgânico) tiveram como melhores dosagens de 15 a 60 e 20 a $60 \mathrm{~g} \mathrm{~m}^{-2}$, respectivamente. Com esta aplicação de regras difusas em dados reais, pode-se levar estes benefícios aos envolvidos na cadeia produtiva do rabanete, resultando assim em uma redução nas dosagens de produtos e melhorando sua rentabilidade final.

Palavras-chave: Lógica nebulosa. Fertilizante mineral. Fertilizante orgânico.

Artigo publicado sob a licença Creative Commons - Atribuição 4.0 Internacional (CC BY 4.0). 


\section{INTRODUÇÃO}

O Produto Interno Bruto (PIB) em 2020, apresentou alta em todos os setores do agronegócio brasileiro, mesmo que os outros setores deste indicador mostraram perdas com a pandemia do COVID-19 (BRASIL, 2021). Dentro do Agronegócio um dos setores que se destacaram foram os grãos e a horticultura, principalmente o rabanete (CEPEA, 2021).

A cultura do rabanete possui um ciclo curto desde a plantio a colheita, isto em relação a outras culturas hortícolas (DA SILVA et al., 2017). Por isso, a aplicação de fertilizantes tanto mineral como orgânico exercem grandes interferências na produtividade desta cultura (GODINHO; GASPAROTTO, 2021), pois a ocorrência de desordens fisiológicas de origem nutricional são fatores que influenciam significativamente a produtividade comercial do rabanete (FERREIRA et al., 2011).

Contudo, os fertilizantes minerais e orgânicos são considerados os custos mais altos em uma produção agrícola (SILVA et al., 2014), com isso, a otimização nos processos deve ser eficaz. A lógica fuzzy é um modelo que pode ser aplicado em diversas áreas, principalmente no agronegócio, tendo como objetivo principal otimizar processos produtivos (GODOY et al., 2020).

As teorias que envolvem os conjuntos e a lógica fuzzy tratam da incerteza e a ambiguidade como determinísticas (CANEPPELE et al., 2021). Onde os pesquisadores da lógica Bayesiana trabalham com probabilidades, os estudiosos da lógica fuzzy visualizam diferentes quantidades de pertinência a eventos que não são prováveis, mas são eventos reais (RIGNEL et al., 2011).

A lógica fuzzy permite aplicar incertezas e ambiguidades em um sistema de regras que expressam uma conclusão não sendo como verdadeira ou falsa, mas sendo verdadeira a determinado grau (CANEPPELE et al., 2021). Sabendo que o grau de certeza é conhecido como grau de pertinência, por isso, a lógica fuzzy trabalha com dados e resultados mais próximos da linguagem não matemática, como exemplo, "pode chover" ou "aquele homem é alto" (PUTTI et al., 2017).

Diante disso, este estudo teve como objetivo desenvolver um modelo baseado em regras difusas para avaliar os efeitos causados pela variação nas dosagens de dois fertilizantes um mineral e um fertilizante orgânico no diâmetro de raiz. 


\section{MATERIAIS E MÉTODOS}

O artigo foi baseado em um experimento à campo real, onde foi aplicado dois fertilizantes no solo, sendo um mineral o adubo 5-10-10, ou seja 5\% de nitrogênio, 10\% de fósforo e 10 de potássio e um orgânico, sendo a compostagem bovina no plantio da cultura do rabanete em 5 dosagens diferentes de cada fertilizante. $O$ experimento foi realizado no Colégio Agrícola Estadual Adroaldo Augusto Colombo. Dados experimentais para modelagem foram descritos por (GODINHO; CANEPPELE, 2019).

Foi desenvolvido um sistema baseado em regras difusas com intuito de encontrar quais as melhores dosagens dos fertilizantes (mineral e orgânico) para obter uma alta produtividade com uma gestão financeira adequada.

Este processador possui uma ou mais entradas, conhecida como input e uma ou mais saídas output, onde foram definidos, conforme apresentado na Figura 1.

Figura 1 - Sistema baseado em regras fuzzy para determinar o diâmetro da raiz do rabanete.

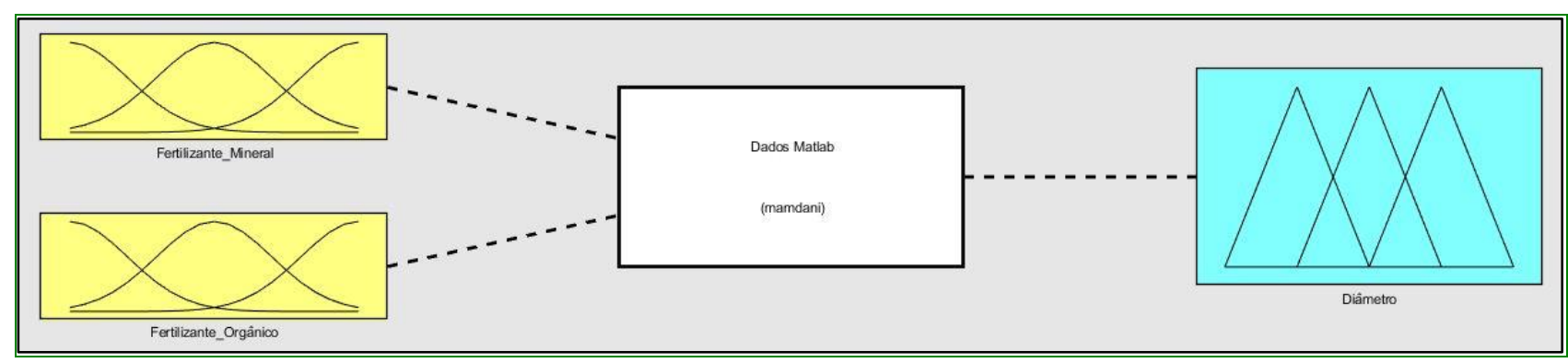

Fonte: Autor, 2021.

Neste estudo, foi realizado um modelo matemático chamado lógica fuzzy para avaliar o diâmetro da raiz do rabanete com as melhores dosagens dos fertilizantes (mineral e orgânico).

As características desta modelagem matemática tem como características uma função onde $f: \mathbb{R}^{2} \rightarrow \mathbb{R}$ com $=f(\bar{x})$, onde $\mathbb{R}$ é o conjunto de números reais, $x_{1}=$ fertilizante mineral, $x_{2}=$ fertilizante orgânico e $y=$ diâmetro, conforme a Figura 1 .

As variáveis de entrada foram o fertilizante mineral (5-10-10) e o fertilizante orgânico (compostagem bovina). De acordo com (TSAl et al., 2020), o modelo mais preciso para este experimento é o trapezoidal.

A equação 1, apresenta o conjunto fuzzy do tipo trapezoidal, que é formado por quatro pontos $(\mathrm{a}, \mathrm{b}, \mathrm{c}, \mathrm{d})$. Portanto, o número de modelagem $(\mathrm{x})$ pode estar contido em 3 
partes do conjunto fuzzy. Assim sendo, sua representação pode ser estabelecida da seguinte forma:

$$
f(x, a, b, c, d)=\left\{\begin{array}{clc}
\frac{x-a}{b-a} & \text { if } & a \leq x<b \\
1 & \text { if } & b \leq x \leq c \\
\frac{d-x}{d-c} & \text { if } & c<x \leq d \\
0 & \text { caso oposto }
\end{array}\right\}
$$

A Figura 2(a) e 2(b) mostram os conjuntos de parâmetros dos fertilizantes (mineral e orgânico), onde foi aplicado intervalo idênticos para ambos de [0 - 60] e foram classificados em "Baixo", "Médio" e "Alto". A Tabela 1 contém os pontos que formam as funções de relevância. Para avaliar o diâmetro da raiz do rabanete, foi utilizado um modelo matemático conforme previamente descrito por (CREMASCO et al., 2010). Por sim, a Figura 2(c) apresenta os valores output para o diâmetro de raiz, onde foi estabelecido um intervalo de $[0-10]$.

Figura 2 - Parâmetro do sistema para a determinação do diâmetro de planta: a) fertilizante mineral; b) fertilizante orgânico; c) diâmetro.

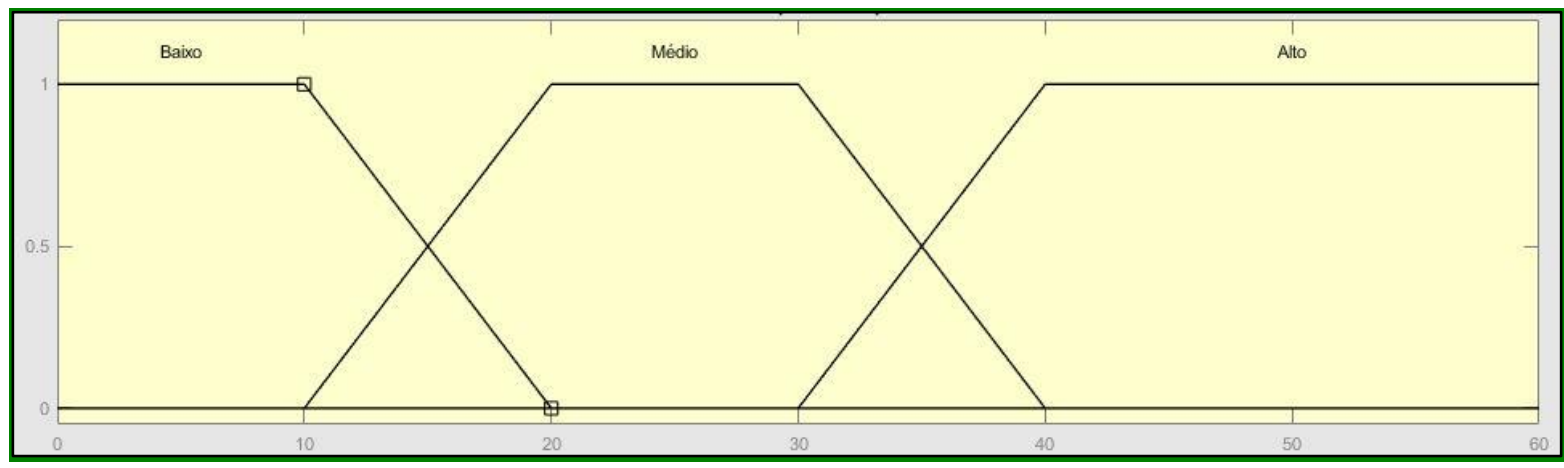

a)

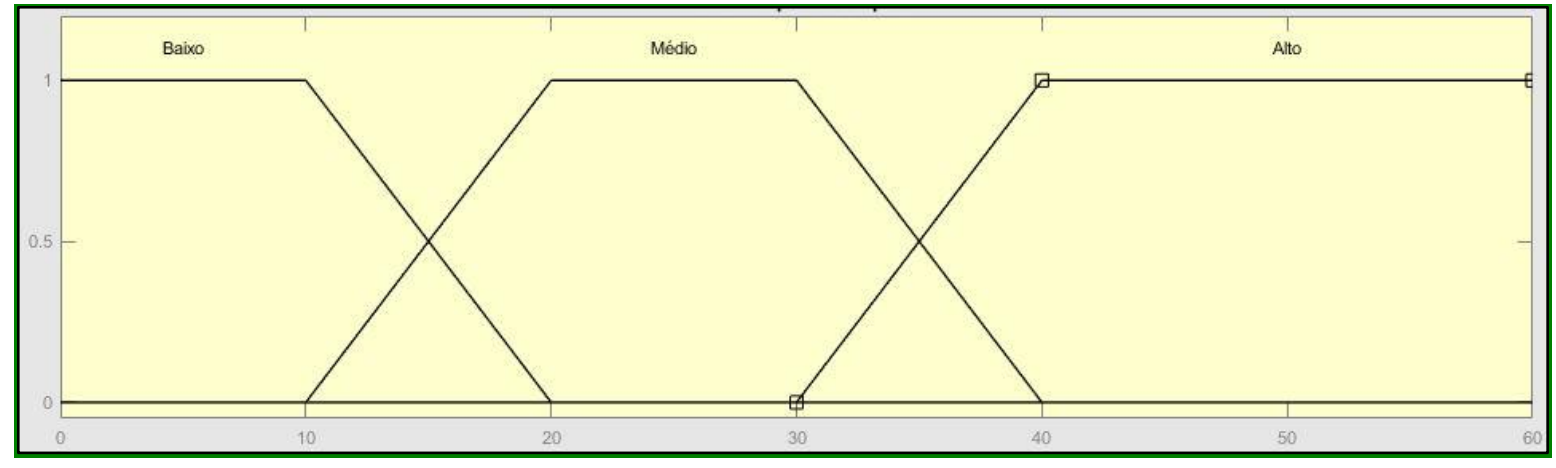

b) 


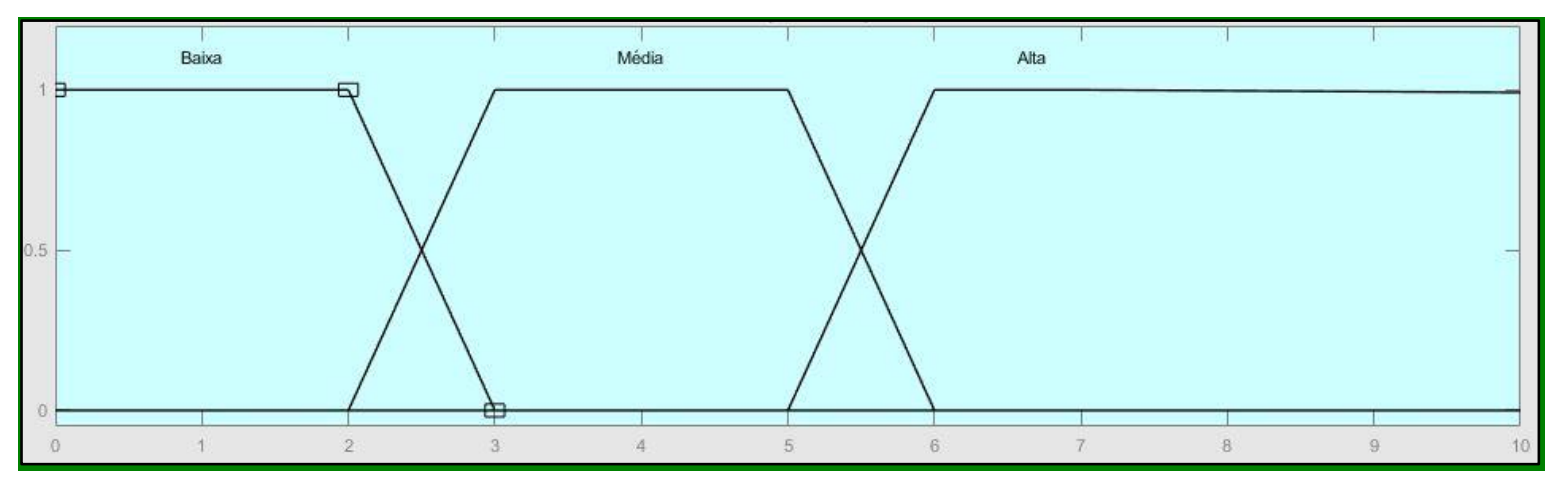

c)

Fonte: Autor, 2021.

Tabela 1 - Definição de funções de relevância para a variável input: fertilizante mineral, fertilizante orgânico e output: diâmetro de raiz do rabanete.

\begin{tabular}{|c|c|c|}
\hline Conjunto difuso & Tipo & Limite \\
\hline \multicolumn{3}{|c|}{ Fertilizante Mineral } \\
\hline "Baixo" B & Trapezoidal & {$\left[\begin{array}{llllll}-1 & 0 & 10 & 20\end{array}\right]$} \\
\hline "Médio" M & Trapezoidal & {$\left[\begin{array}{lllll}10 & 20 & 30 & 40\end{array}\right]$} \\
\hline "Alto" A & Trapezoidal & {$\left[\begin{array}{lllll}30 & 40 & 50 & 60\end{array}\right]$} \\
\hline \multicolumn{3}{|c|}{ Fertilizante Orgânico } \\
\hline "Baixo" B & Trapezoidal & {$\left[\begin{array}{lllll}-1 & 0 & 10 & 20\end{array}\right]$} \\
\hline "Médio" M & Trapezoidal & {$\left[\begin{array}{lllll}10 & 20 & 30 & 40\end{array}\right]$} \\
\hline "Alto" A & Trapezoidal & {$\left[\begin{array}{llll}30 & 40 & 50 & 60\end{array}\right]$} \\
\hline \multicolumn{3}{|c|}{ Diâmetro } \\
\hline "Baixo" B & Trapezoidal & {$\left[\begin{array}{lllll}-1,0 & 0 & 1,0 & 2,0\end{array}\right.$} \\
\hline "Médio" M & Trapezoidal & {$[1,02,03,04,0]$} \\
\hline "Alto" A & Trapezoidal & {$\left[\begin{array}{llllll}3,0 & 4,0 & 5,0 & 6,0\end{array}\right.$} \\
\hline
\end{tabular}

Fonte: Autor, 2021.

O sistema foi baseado em regras difusas de computador foi estabelecido pela Fuzzy Logic Toolbox de MATLAB® 7.0 (MathWorks Inc. Copyright 1984-2004), acoplado à superfície e do mapa de contorno.

\section{RESULTADOS E DISCUSSÃO}

A partir do experimento de campo com o rabanete, foi possível traçar a regra base (Tabela 2). Assim, o diâmetro da raiz do rabanete tende a responder com altas produtividades, quando a sua adubação está em níveis adequados de nutrientes. 
Tabela 2 - Regras básicas do sistema fuzzy.

\begin{tabular}{|c|c|c|}
\hline Fertilizante Mineral & Fertilizante Orgânico & Diâmetro (cm) \\
\hline$B$ & $B$ & $B$ \\
\hline $\mathrm{B}$ & $M$ & $\mathrm{~B}$ \\
\hline $\mathrm{B}$ & A & $\bar{M}$ \\
\hline$M$ & $B$ & $B$ \\
\hline$M$ & $\mathrm{M}$ & $\mathrm{M}$ \\
\hline $\mathrm{M}$ & $A$ & $\mathrm{~A}$ \\
\hline$A$ & $B$ & $\mathrm{M}$ \\
\hline$A$ & $M$ & $A$ \\
\hline$A$ & $A$ & A \\
\hline
\end{tabular}

B: BOM; M: MÉDIO; A: ALTO

Fonte: Autor, 2021.

A Tabela 2, apresenta as regras básicas do sistema, onde foi elaborado a partir de dados linguísticos da lógica fuzzy. Sendo:

- Se (fertilizante mineral é "B") (fertilizante orgânico é "B") então (diâmetro é "B");

- Se (fertilizante mineral é "B") (fertilizante orgânico é "M") então (diâmetro é "B");

- Se (fertilizante mineral é "B") (fertilizante orgânico é "A") então (diâmetro é "M");

- As outras linhas são interpretadas de forma análoga.

A partir do modelo desenvolvido, foi possível preparar a resposta das superfícies para o diâmetro da raiz e seus mapas de contorno para verificar a real inferência dos fertilizantes mineral e orgânico. $O$ modelo baseado em regras fuzzy verificou todas as combinações entre as variáveis, sendo 3 níveis (B; M; $A$ ) com 2 variáveis (fertilizante mineral e fertilizante orgânico) pode criar uma base de regras com 9 combinações, sendo $3 \times 3$.

Com isso, foi desenvolvido o modelo de superfície de resposta difusa na Figura 3. 
Figura 3 - Modelo de superfície de resposta do diâmetro de raiz do rabanete em resposta aos fertilizantes mineral e orgânico.

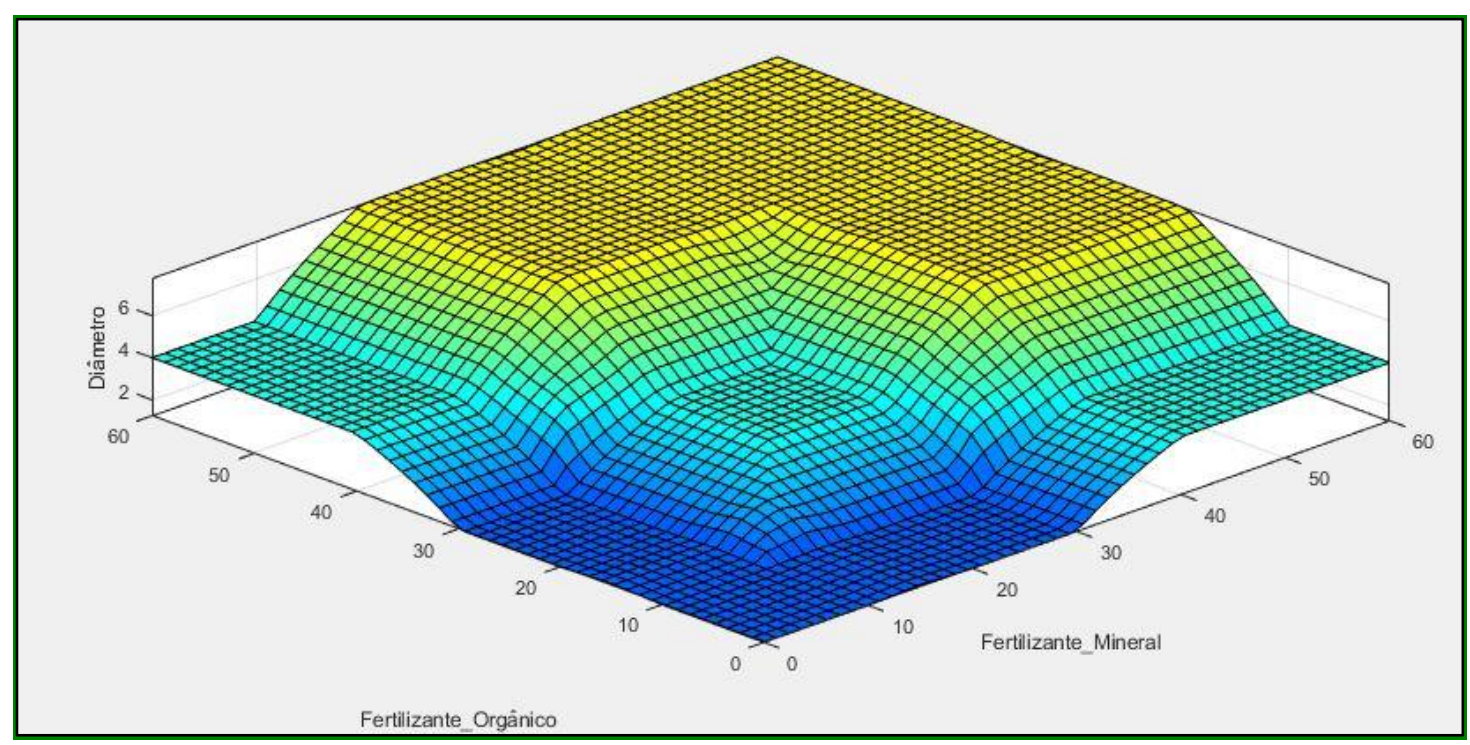

Fonte: Autor, 2021.

Neste estudo, foi observada uma forte relação entre a aplicação do fertilizante mineral e do fertilizante orgânico determinando o diâmetro de raiz do rabanete. Em outras palavras, o rabanete responde bem a aplicações de fertilizantes de média a altas dosagens para altas produtividades, conforme a apresentada na Figura 3.

A Figura 4 é o mapa de contorno produzido após a aplicação das bases de regras desenvolvidas pela lógica fuzzy. 
Figura 4 - Mapa de contorno do diâmetro de raiz do rabanete em resposta aos fertilizantes mineral e orgânico.

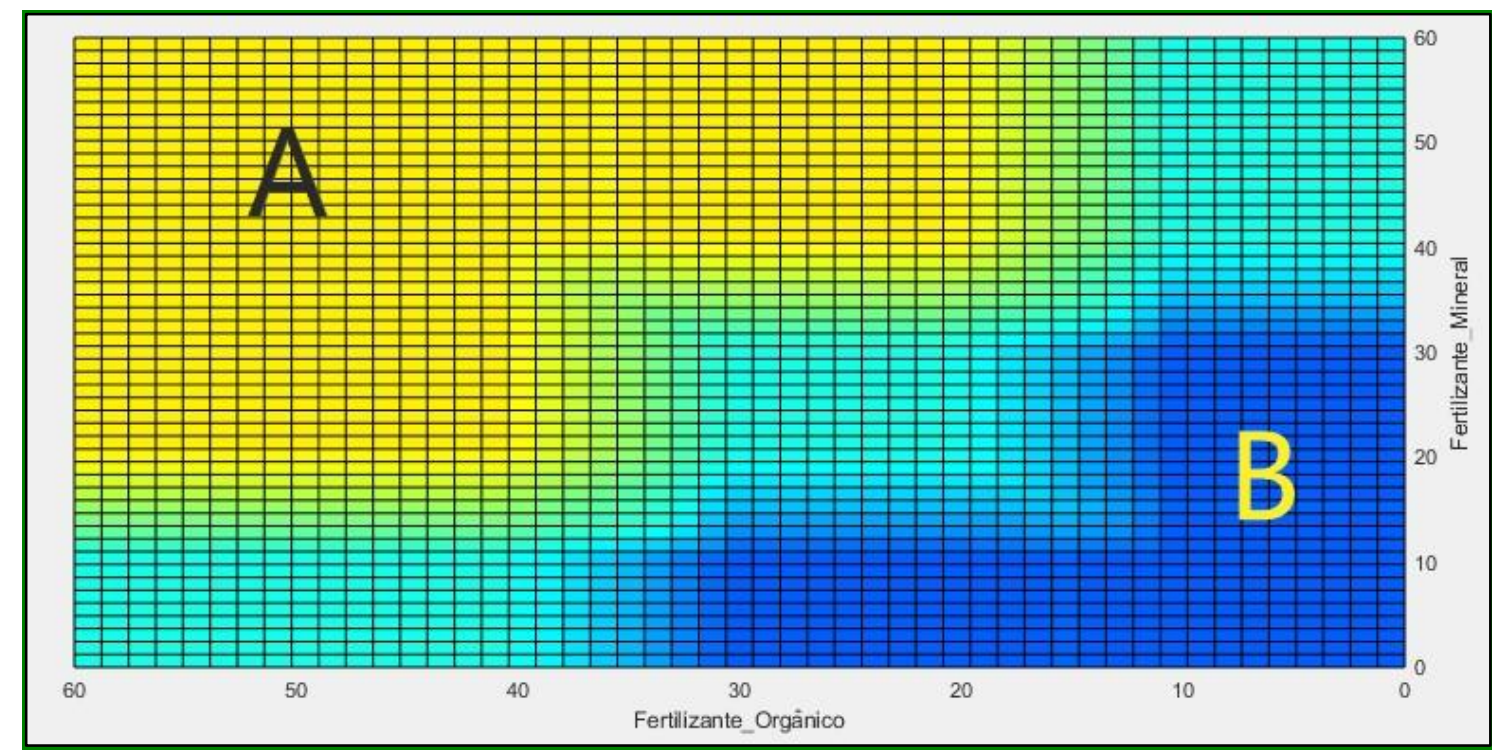

Fonte: Autor, 2021.

A região B ilustrada na Figura 4 representa uma condição de diâmetro de raiz desfavorável devido à baixa dosagem dos fertilizantes, principalmente pelo orgânico, o que não proporcionam as condições ideais para o crescimento e desenvolvimento desta cultura específica. Por outro lado, a região A é caracterizada por ser uma área que apresenta taxas médias a altas para o fertilizante mineral e taxas médias a altas para o orgânico, proporcionando uma condição de região adequada para altas produtividades do rabanete.

Em um experimento a campo com diferentes dosagens de fertilizantes orgânicos FO, em hortaliças, (GODINHO et al., 2019) confirmam que em altas dosagens de FO, as hortaliças em geral tendem a responder com maior aptidão para produtividades, pois nestes fertilizantes além de possuir nutrientes em sua constituição, também possui altas taxas de matéria-orgânica.

A Figura 5 simula o sistema com base em regras difusas para as dosagens dos fertilizantes mineral e orgânico. 
Figura 5 - Método de inferência de Mandani para fertilizante mineral $=30 \mathrm{~g} \mathrm{~m}^{-2}$ e fertilizante orgânico $=30 \mathrm{~g}$ $\mathrm{m}^{-2}$, com um diâmetro de raiz difusa $=4,0$.

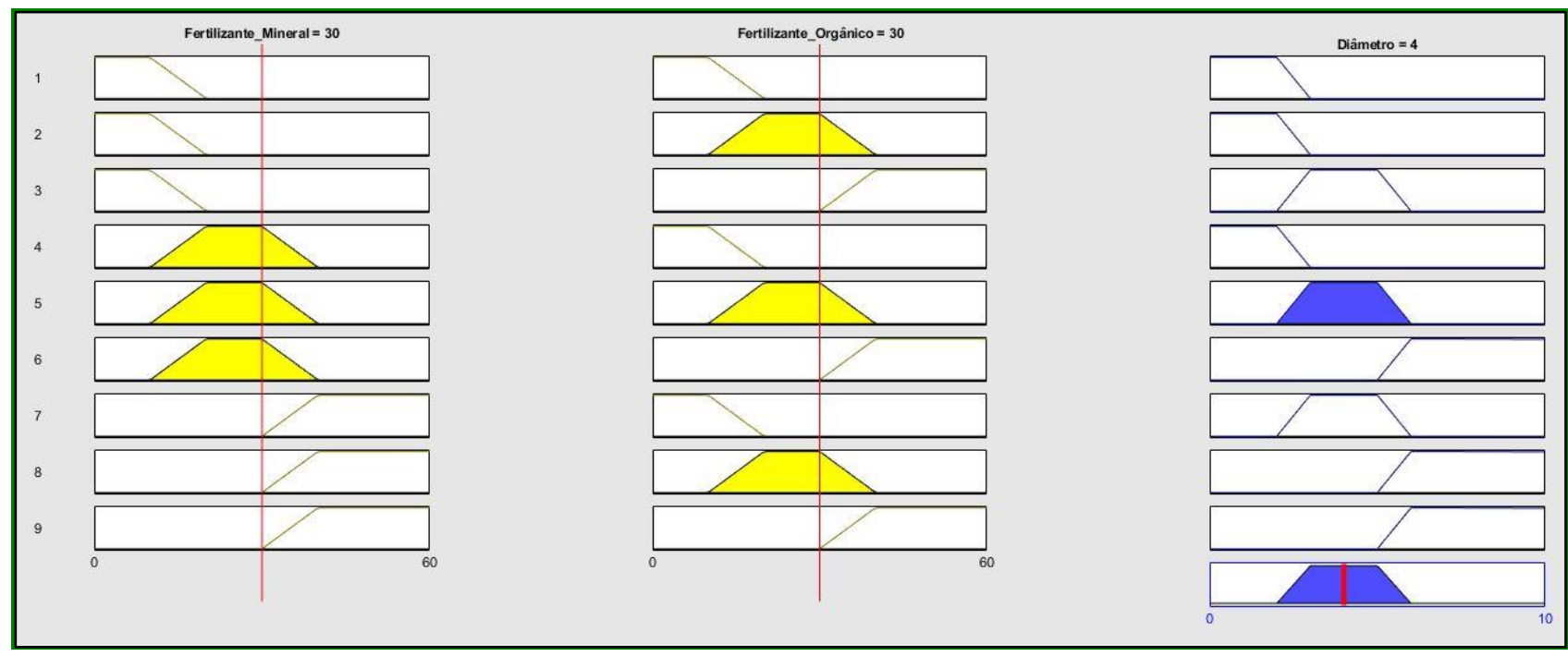

Fonte: Autor, 2021.

O ponto encontrado está dentro de uma condição intermediária, que também é levado em consideração para determinar o diâmetro de raiz, dada como $4,0 \mathrm{~cm}$. Quando os graus analisados da associação da variável saída são analisados, percebe-se que o ponto possui maior grau de pertinência dentro o conjunto difuso, como mostra a Figura 6 .

Figura 6 - Indicação de maior grau de pertinência para o conjunto difuso, ponto ótimo de diâmetro de raiz $=4,0$.

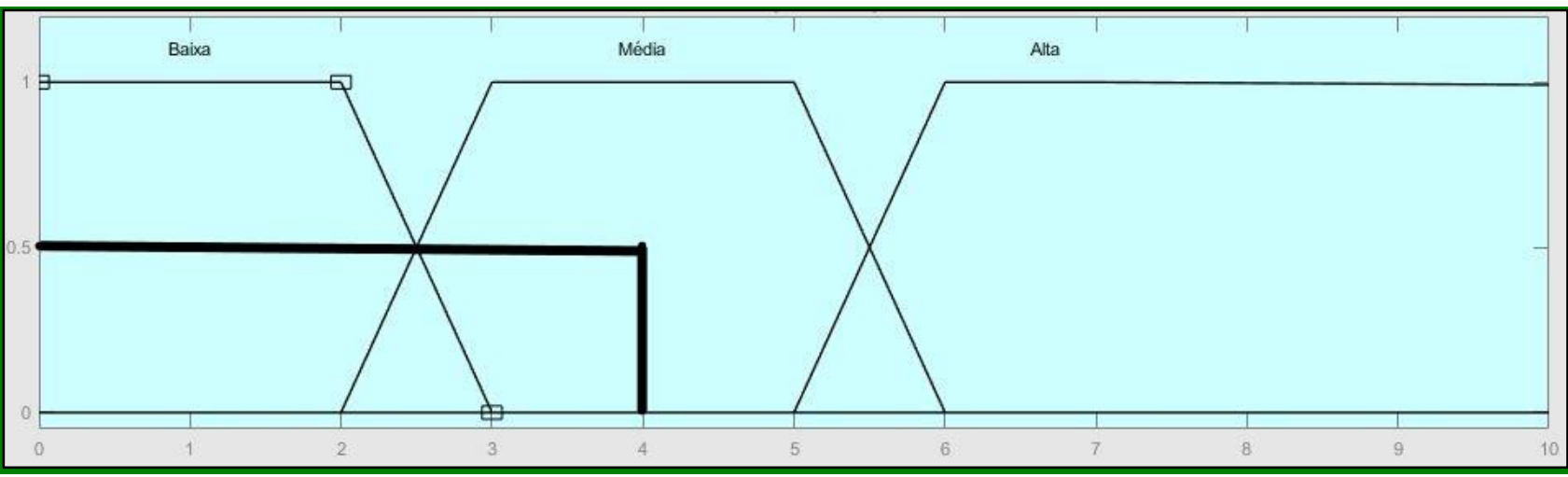

Fonte: Autor, 2021. 


\section{CONCLUSÕES}

A interpretação do uso da lógica fuzzy neste experimento utilizando dados reais de campo conseguiu otimizar as melhores dosagens dos fertilizantes utilizados, sendo 15 a 60 e 20 a $60 \mathrm{~g} \mathrm{~m}^{-2}$, respectivamente do fertilizante orgânico e do fertilizante mineral.

Com esse resultado, o produtor de hortaliças principalmente de rabanete, pode otimizar as dosagens dos fertilizantes tanto mineral como orgânico em seus canteiros, com maior eficiência e eficácia buscando maior rentabilidade financeira.

\section{REFERÊNCIAS}

BRASIL. Produto Interno Bruto - PIB. Disponível em: https://www.gov.br/fazenda/ptbr/centrais-de-conteudos/publicacoes/conjuntura-economica/atividadeeconomica/2021/pib-brasil-4tri2020-ibge-resultados-spe.pdf. Acesso em: 26.05.2021.

CANEPPELE, F. de L.; GODINHO, E. Z.; ZUIN, L. F. S.; GABRIEL FILHO, L. R. A. Aplicação da lógica fuzzy no desenvolvimento do morango no oeste do Paraná. Revista Sodebras, São Paulo, São Paulo, Brasil, v. 16, n. 184, p. 2019-2022, 2021. DOI:

\section{CEPEA. PIB do agronegócio alcança participação de $\mathbf{2 6 , 6 \%}$ no PIB brasileiro em} 2020. Piracicaba: 2021. Disponível em: https://www.cnabrasil.org.br/boletins/pib-doagronegocio-alcanca-participacao-de-26-6-no-pib-brasileiro-em-2020. Acesso em: 26.05.2021.

CREMASCO, C. P.; ROBERTO, L.; GABRIEL, A. metodologia de determinação de funções de pertinência de controla- dores fuzzy para a avaliação energética de empresas de avicultura de postura. Revista Energia na Agricultura, Botucatu, São Paulo, Brasil, v. 25, n. 1, p. 21-39, 2010. DOI: 10.17224/EnergAgric.2010v25n1p21-39.

DA SILVA, A. F. A. et al. Desempenho agronômico do rabanete adubado com Calotropis procera (Ait.) R. Br. em duas épocas de cultivo. Revista Ciência Agronômica, Fortaleza, Ceará, Brasil, v. 48, n. 2, p. 328-336, 2017.

FERREIRA, R. L. F. et al. Produção orgânica de rabanete em plantio direto sobre cobertura morta e viva. Horticultura Brasileira, Brasília, Distrito Federal, Brasil, v. 29, n. 1, p. 299-303, 2011.

GODINHO, E. Z. et al. Resposta da beterraba à aplicação de fertilizante mineral e organomineral no cultivo de verão. Revista Agropampa, Porto Alegre, Rio Grande do Sul, Brasil, v. 2, n. 2, p. 238-248, 2019.

GODINHO, E. Z.; CANEPPELE, F. DE L. Efeito da nutrição. Revista Cultivar - grandes culturas, Curitiba, Paraná, Brasil, v. 1, n. 241, p. 20-23, 2019. 
GODINHO, E. Z.; GASPAROTTO, H. V. Resposta da cenoura ao uso de diferentes fertilizantes de solo. Brazilian Journal of Development, São José do Pinhais, Paraná, Brasil, v. 7, n. 3, p. 27052-27059, 2021. DOl: 10.34117/bjdv7n3-420.

GODOY, F. O. DE et al. Utilização da lógica fuzzy aplicada à energia solar. Cadernos de Ciência \& Tecnologia, Brasília, Distrito Federal, Brasil, v. 37, n. 2, e26663, 2020. DOI: 10.35977/0104-1096.cct2020.v37.26663.

PUTTI, F. F. et al. A Fuzzy mathematical model to estimate the effects of global warming on the vitality of Laelia purpurata orchids. Mathematical Biosciences, v. 288, p.124-129, 2017.

RIGNEL, D. G. D. S.; CHENCI, G. P.; LUCAS, C. A. Uma Introdução a Lógica Fuzzy. Revista Eletrônica de Sistemas de Informação e Gestão Tecnológica, Franca, São Paulo, Brasil, v. 1, n. 1, p. 17-28, 2011.

SILVA, P. P. DA et al. Resposta de cultivares de Brachiaria brizantha a doses de biofertilizantes de aves. Arquivos do Instituto Biológico, São Paulo, São Paulo, Brasil, v. 81, n. 3, p. 286-289, set. 2014. DOI: 10.1590/1808-1657001282012

TSAI, W. T. et al. Conversion of water caltrop husk into torrefied biomass by torrefaction. Energy, v. 195, e116967, 2020. 


\begin{abstract}
The aim of this study was to develop a fuzzy-rules-based model to evaluate the effects caused by varying the dosages of two soil fertilizers (mineral and organic) on root diameter. Fuzzy logic is a method that presents a language, more appropriate to everyday life, as the sky is cloudy. For the input variables of this system, mineral and organic fertilizers were used, for the output the root diameter, in $\mathrm{cm}$. After optimizing the input rules, it can be seen that for the application of fertilizers (mineral and organic) the best dosages were 15 to 60 and 20 to $60 \mathrm{~g} \mathrm{~m}-2$, respectively. With this application of fuzzy rules in real data, these benefits can be taken to those involved in the radish production chain, thus resulting in a reduction in product dosages and improving its final profitability.
\end{abstract}

Keywords: Fuzzy logic. Mineral fertilizer. Organic fertilizer.

\title{
RESUMEN
}

El objetivo de este estudio fue desarrollar un modelo basado en reglas difusas para evaluar los efectos causados por la variación de las dosis de dos fertilizantes de suelo (mineral y orgánico) sobre el diámetro de la raíz. La lógica difusa es un método que presenta un lenguaje, más apropiado para la vida cotidiana, ya que el cielo está nublado. Para las variables de entrada de este sistema se utilizaron fertilizantes minerales y orgánicos, para la salida el diámetro de la raíz, en $\mathrm{cm}$. Luego de optimizar las reglas de entrada, se puede observar que para la aplicación de fertilizantes (minerales y orgánicos) las mejores dosis fueron de 15 a 60 y de 20 a $60 \mathrm{~g} \mathrm{~m}^{-2}$, respectivamente. Con esta aplicación de reglas difusas en datos reales, estos beneficios se pueden llevar a los involucrados en la cadena de producción del rábano, resultando así en una reducción en las dosis del producto y mejorando su rentabilidad final.

Palabras-clave: Lógica difusa. Fertilizante mineral. Fertilizante orgánico. 


\section{LICENÇA DE USO}

Este é um artigo publicado em acesso aberto (Open Access) sob a licença Creative Commons Atribuição 4.0 Internacional (CC BY 4.0), que permite uso, distribuição e reprodução em qualquer meio, desde que o trabalho original seja corretamente citado. Mais informações em: http://creativecommons.org/licenses/by/4.0

\section{CONFLITO DE INTERESSES}

Os autores declaram que não há conflito de interesses neste trabalho.

\section{CONTRIBUIÇÕES AUTORAIS}

Autor 1: Montagem do experimento à campo de onde foi coletado os dados, também montou o artigo no modelo da revista, aplicou os dados no software, fez os resultados e discussão e a conclusão.

Autor 2: Supervisão geral do artigo, correções gerais.

Autor 3: Organização do artigo na questão da introdução, tradução do resumo para o inglês e revisão do artigo.

\section{FINANCIAMENTO}

O presente trabalho não contou com apoio financeiro.

\section{COMO REFERENCIAR}

GODINHO, Emmanuel Zullo; CANEPPELE, Fernando de Lima; GASPAROTTO, Hélio Vagner. Utilização da lógica fuzzy para otimizar aplicação de fertilizantes no rabanete. Revista Brasileira de Engenharia de Biossistemas (Tupã), v. 15, n. 2, p. 270-282, 2021. DOI: http://dx.doi.org/10.18011/bioeng2021v15n2p270-282

\section{RESPONSABILIBADE EDITORIAL}

Prof. Dr. Fernando Ferrari Putti ${ }^{1}$, Prof. Dr. Paulo Sérgio Barbosa dos Santos ${ }^{1}$, Prof. Dr. Eduardo Festozo Vicente ${ }^{1}$ e Prof. Dr. Diogo de Lucca Sartori ${ }^{1}$

${ }^{1}$ Universidade Estadual Paulista "Júlio de Mesquita Filho", FCE - Faculdade de Ciências e Engenharia, Tupã, SP, Brasil. 